\title{
Review \\ Blue Phase Liquid Crystals with Tailored Crystal Orientation for Photonic Applications
}

\author{
SeongYong Cho and Masanori Ozaki *
}

check for updates

Citation: Cho, S.; Ozaki, M. Blue Phase Liquid Crystals with Tailored Crystal Orientation for Photonic Applications. Symmetry 2021, 13, 1584. https: / /doi.org/10.3390/ sym13091584

Academic Editors: Shoichi Ishihara and Sadahito UTO

Received: 21 July 2021

Accepted: 23 August 2021

Published: 27 August 2021

Publisher's Note: MDPI stays neutral with regard to jurisdictional claims in published maps and institutional affiliations.
Division of Electrical, Electronic and Infocommunication Engineering, Graduated School of Engineering, Osaka University, 2-1 Yamada-Oka, Suita, Osaka 565-0871, Japan; scho@opal.eei.eng.osaka-u.ac.jp

* Correspondence: ozaki@eei.eng.osaka-u.ac.jp

\begin{abstract}
Blue phase (BP) liquid crystals, which self-assemble into soft three-dimensional (3D) photonic crystals, have attracted enormous research interest due to their ability to control light and potential photonic applications. BPs have long been known as optically isotropic materials, but recent works have revealed that achieving on-demand 3D orientation of BP crystals is necessary to obtain improved electro-optical performance and tailored optical characteristics. Various approaches have been proposed to precisely manipulate the crystal orientation of BPs on a substrate, through the assistance of external stimuli and directing self-assembly processes. Here, we discuss the various orientation-controlling technologies of BP crystals, with their mechanisms, advantages, drawbacks, and promising applications. This review first focuses on technologies to achieve the uniform crystal plane orientation of BPs on a substrate. Further, we review a strategy to control the azimuthal orientation of BPs along predesigned directions with a uniform crystal plane, allowing the 3D orientation to be uniquely defined on a substrate. The potential applications such as volume holograms are also discussed with their operation principle. This review provides significant advances in 3D photonic crystals and gives a huge potential for intelligent photonic devices with tailored optical characteristics.
\end{abstract}

Keywords: blue phase liquid crystals; photonic crystals; 3D orientation control; photonic devices

\section{Introduction}

Blue phase (BP) liquid crystals (LCs), which are a kind of chiral LC state, have emerged significant research interest in a variety of photonic applications owing to their threedimensional (3D) photonic structures. They are built by self-assembling so-called double twist cylinders (DTCs) into two types of cubic symmetry: body-centered cubic (BP I, I4 ${ }_{1} 32$ ) and simple cubic (BP II, $\mathrm{P}_{2} 32$ ), depending on the chiral twisting power and temperature (Figure 1) [1-3]. The highly ordered structures of BPs give rise to a unique selective Bragg reflection in the visible light region, which only reflect a circular-polarized (CP) light with the same handedness as the helix $[4,5]$. The central wavelength of the selective Bragg reflection $\left(\lambda_{c}\right)$ is expressed as

$$
\lambda_{c}=\frac{2 n a}{\sqrt{h^{2}+k^{2}+l^{2}}} \cos \theta
$$

where $n$ is the average refractive index of BPs, $a$ is the lattice constant of BPs, $(h k l)$ is the Miller indices representing the crystal orientation, and $\theta$ is the propagation angle of light in BPs with respect to the normal direction of a Miller plane. Because of the external stimuli-responsivity of BPs, they can be easily switched and tuned by controlling temperature [6,7], by applying an electric field [7-11], and by illuminating light [12,13], making them applicable to various photonic applications such as displays [14], photonic crystal lasers [6,15-18], biosensors [19,20], optical filters [21], polarization converters [22], and diffractive optics with the capability of nonmechanical beam steering $[23,24]$. 
(a)

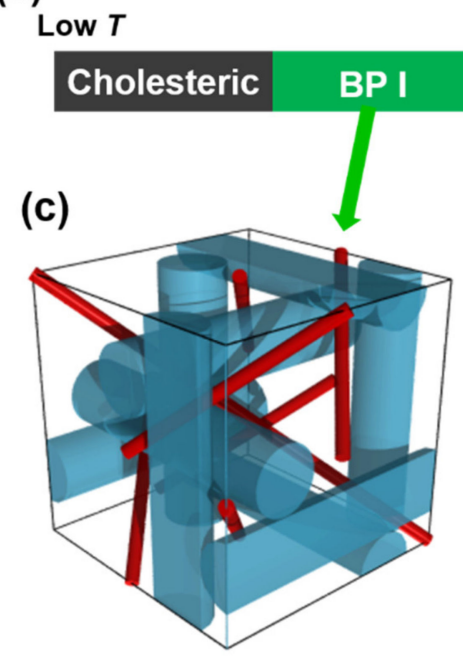

Body-centered cubic
High $T$

(b)

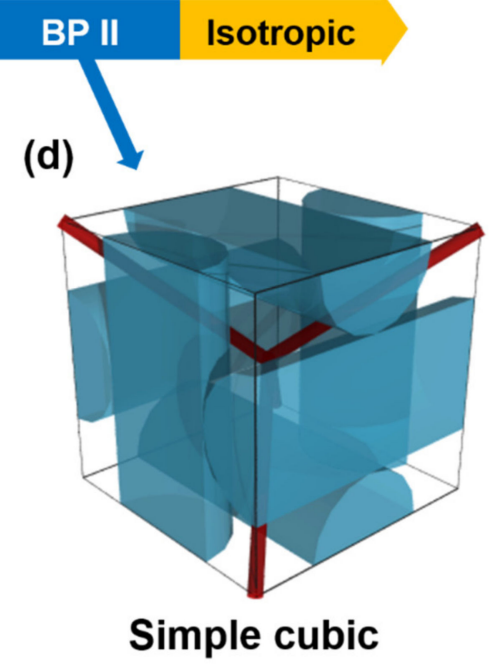

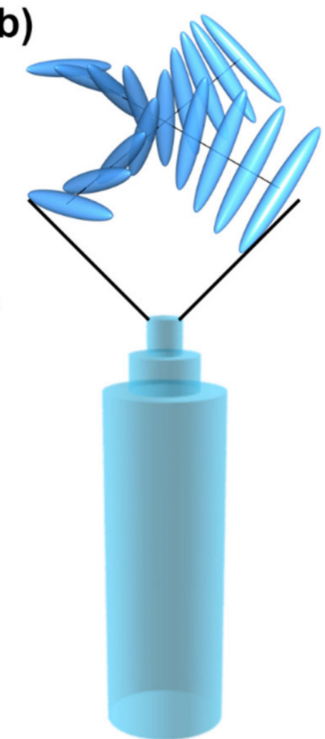

Figure 1. (a) Temperature range in which BP I and BP II appear. (b) Structure of a double-twist cylinder (DTC) composed of biaxial twist of the LC molecules. (c,d) Unit structures of body-centered cubic BP I (c) and simple cubic BP II (d), with their dislocation lines (red lines).

Despite the technological potential of BPs, several issues have been raised to achieve tailored light-matter interaction for practical applications. First, BPs typically appear in a narrow temperature range of approximately $1-2{ }^{\circ} \mathrm{C}$ between cholesteric $(\mathrm{Ch})$ and isotropic state. BPs that exhibit over a wide range of temperature were obtained by adding a small amount of photo-reactive monomers and in situ polymerization, providing a temperature range of BPs over $50{ }^{\circ} \mathrm{C}$ including room temperature $[25,26]$. Other strategies have also been proposed to extend the temperature range of BPs, by dispersing nanoparticles [27,28], bent-core molecules [6,29], and T-shaped molecules [30], by confining BPs in the microsized walls [31-33], and by designing the chemical structure of LC molecules [34]. Second, cubic BPs generally exhibit polycrystalline structures, which are composed of small platelet domains with different crystallographic orientations. Because of the complicated structures of BPs, it is not a trivial task to achieve the uniform crystal orientation in the entire device area. However, BPs with a uniform crystal plane orientation have shown superior optical characteristics compared to multidomain BPs [35]. Moreover, it was found that BPs provide a way to arbitrarily steer the wavefront of reflected light by azimuthally rotating the 3D crystals [36], enabling us to fabricate various diffractive optical elements (DOEs), such as light deflectors [36] and holograms [37]. Thus, it is necessary to accomplish the tailored 3D orientation of $\mathrm{BP}$ crystals from the practical perspective.

In this review, we focus on recent developments to effectively produce monodomain BPs in which a specific Miller plane is uniformly oriented parallel to a substrate. Various approaches such as the assistance of external stimuli and directing the self-assembly processes are discussed with the designs, mechanisms, and potential applications. While the approaches allow monodomain BPs in a large area, they still exhibit grain boundaries between the domains. An approach that realizes macroscopic single-crystal BPs using nanopatterned templates is also discussed. Further, in contrast to the technologies that allow only crystal plane orientation to be manipulated, we discuss a new strategy to simultaneously control two crystal axes of BP I on a substrate. The method, which is referred to as field-assisted directed self-assembly process, enables us to obtain a BP I with the [110] crystal axis oriented perpendicular to the substrate and the [001] crystal axis aligned along the predesigned surface anchoring. The mechanism with its potential applications such as volume holograms is presented. In the following, we refer to the BP I 
and BP II with the ( $h k l)$ crystal plane oriented parallel to the substrate as BP $\mathrm{I}_{(h k l)}$ and $\mathrm{BP} \mathrm{II}_{(h k l)}$, respectively.

\section{Crystal Plane Orientation-Controlling Technologies}

In many researches, BPs are regarded as an isotropic material with scalar electro-optic properties, thus less attention has been paid to the importance of controlling the crystal orientation. However, considering that the wavelength of Bragg reflection depends on the Miller planes (according to Equation (1)) and small platelet domains greatly degrade the performance of BP-based optical devices, it is necessary to obtain the tailored 3D orientation of BP crystals on a substrate. Belyakov et al. revealed that the optical rotatory power of a single-crystal BP is significantly enhanced compared to the polycrystalline BPs [5]. Moreover, enlarging the domain size of polymer-stabilized BPs (PSBPs) enables hysteresisfree electro-optical switching due to the reduced grain boundaries between the different crystal orientations [38]. Thus, controlling the crystal orientation and understanding the mechanisms are important from the fundamental and practical perspective. In the following, we discuss recent developments to control the orientation of BP crystals over a large area, including thermal control, the use of alignment layers, electric field treatment, and the use of nanopatterned templates. It should be noted that we refer to BPs consisting of small crystalline platelets with a uniform crystal plane orientation as monodomain BPs, which still have grain boundaries between the platelets. The single-crystal BPs indicate those consisting of a uniform crystal plane and an azimuthal orientation without the grain boundaries.

\subsection{Thermal Control}

Chen et al. investigated a thermal recycling process to obtain enlarged domains of BPs with a uniform crystal orientation [38]. By repeatedly performing the cooling and heating process in a temperature range where BP I appears, the heterogeneous nucleation (nucleation occurring at substrate surface) becomes dominant over the homogeneous nucleation (nucleation occurring in the bulk) during the crystal growth. As a result, the uniform BP $\mathrm{I}_{(220)}$ was obtained with a domain size enlarged by approximately five times compared to the initial state. The uniform BP I showed significantly improved electro-optical hysteresis and higher transmittance due to the reduced light scattering, demonstrating the capability of using them as photonic applications such as displays and tunable LC lenses.

Extremely large monocrystalline BPs were attained by controlling the self-assembly process of BPs [39]. A LC mixture was cooled from the isotropic state to a temperature where BP I and BP II appear, and held for ten hours to induce the reassembly of the BP platelets, as shown in the polarized optical microscope (POM) images in Figure 2a,b. Interestingly, as a result of observing the crystal growth of BPs over time, only the domains of the BP II were reassembled and grown larger, whereas no significant change was observed in the domains of the BP I. This originates from the topological differences of the lattice structure and dislocation lines between BP I and BP II. In a unit cell of BP II, all dislocation lines are intersected at the center of the unit cell, playing as a single defect. Thus, there exists only one minimum free energy for the formation of a monocrystal. On the other hand, the BP I makes it difficult to form a monocrystal because the dislocation lines that are separated from each other have many minimum free energies. Despite the difficulty of forming a large BP I domain using a direct self-reassembly process, two approaches were proposed: using a well-aligned BP II as a precursor for monocrystal BP I and a gradient-temperature scanning (GTS) technique. A BP I with approximately $\mathrm{mm}^{2}$-sized monodomains was obtained by rapidly cooling from enlarged BP II domains and holding the temperature for $24 \mathrm{~h}$. Further, for the GTS technique, the monocrystal BP I placed on a low $T$-stage was exploited as a seed for nucleation (Figure 2c). When pushing the sample towards the low T-stage, BP I was grown from the seed with a uniform crystal plane orientation (Figure 2d). By adjusting the pushing direction two-dimensionally, a BP 
crystal having the size of $1200 \times 700 \mu \mathrm{m}^{2}$ with a thickness of $\sim 300 \mu \mathrm{m}$ was obtained. The monocrystal BP I showed improved Bragg reflectivity, narrow bandwidth, and electrical tunability over a wide range of wavelengths $(\sim 100 \mathrm{~nm})$, demonstrating its ability as versatile 3D photonic crystal devices.

(a)

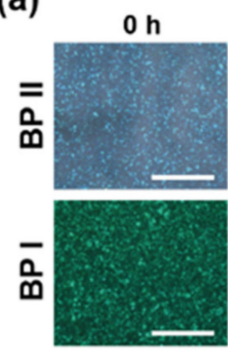

$2 \mathrm{~h}$
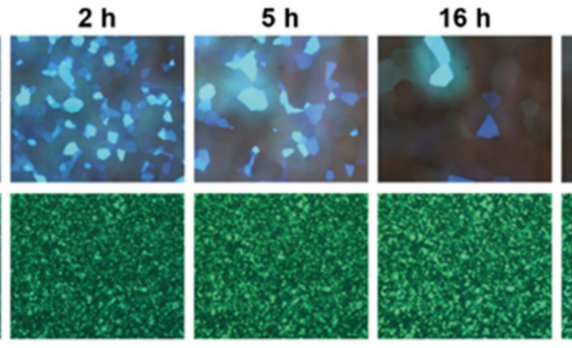

(c)

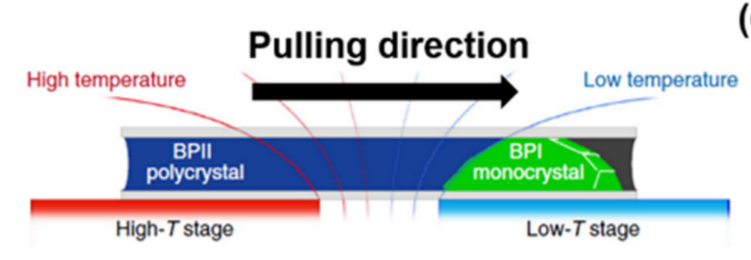

(b)
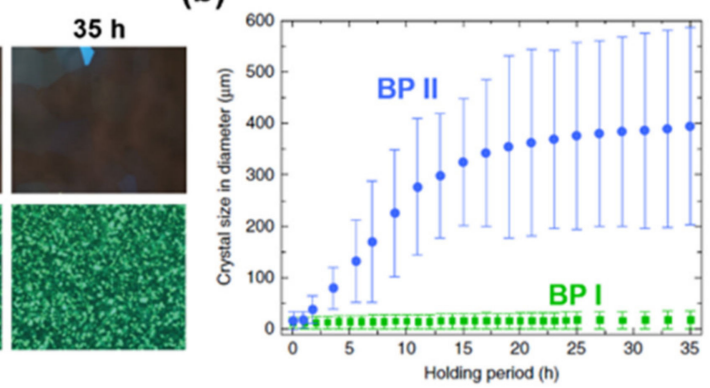

(d)

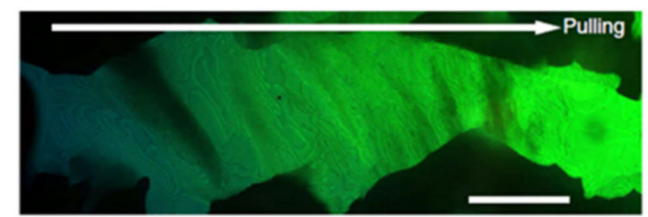

Figure 2. (a) Time evolutions of microscopic images of BP II (upper) and BP I (bottom) crystals. (b) Average domain size of BP II and BP I at various holding times. (c) Experimental description to obtain large single-crystal BP I through gradient-temperature scanning (GTS) method. (d) Polarized optical microscope (POM) image of single-crystal BP I obtained by GTS method. Reproduced with permission from [39]. Copyright 2017, Nature Publishing Group.

It is possible to obtain enlarged domains of BPs using the aforementioned methods, but only a slow rate of temperature control ensures the crystal orientation to be effectively controlled. Thus, they are all time-consuming and require complex equipment to precisely control the temperature.

\subsection{Use of the Alignment Layer}

One of the simplest methods to obtain monodomain BPs is to coat an appropriate alignment layer on a substrate and imprint the orientational easy axis of the LC director. Many researchers have obtained monodomain BPs particularly using a rubbing treatment and demonstrated that they possess better electro-optical and photonic characteristics than multidomain BPs. For example, Nayek et al. fabricated a monodomain BP using an antiparallel rubbed cell, which showed a reduced operating voltage by $27 \%$ and hysteresis by $63 \%$ due to the enhanced Kerr constant [40]. Similarly, a well-aligned BP II was obtained by cooling from an isotropic state in a rubbed cell, providing significantly improved reflectivity with a narrow photonic band gap [41]. A polarization-independent phase modulator was also developed using monodomain PSBPs [42]. The monodomain PSBPs showed a maximum phase shift higher than multidomain PSBPs by a factor of 1.5 under a certain applied field. A well-aligned PSBP II has shown that the hysteresis for electro-optical switching was drastically reduced compared to the multidomain PSBP I [43].

Further advanced capabilities of monodomain BPs as photonic applications were also revealed. The threshold energy for laser emission from the well-aligned BP II was drastically reduced compared to multidomain BPs, as well as the full-width of half-maximum (FWHM) of the emission became narrower by four times (Figure 3a-d) [18]. The reversibly tunable emission wavelength over a range of $40 \mathrm{~nm}$ was realized by adjusting the lattice constant of the well-aligned BP II through the thermal control.

A diffraction grating was demonstrated by inducing the local reorientation of the LC director through the in-plane electric field (Figure 3e). The electric field induced the LC 
birefringence owing to the Kerr effect, causing spatial modulation of the refractive index. Because the regular arrangement of the monodomain BPs has a long coherence length, the driving voltage was reduced by $29 \%$ with the improved diffraction efficiency by $9 \%$ (Figure 3f,g) [44].
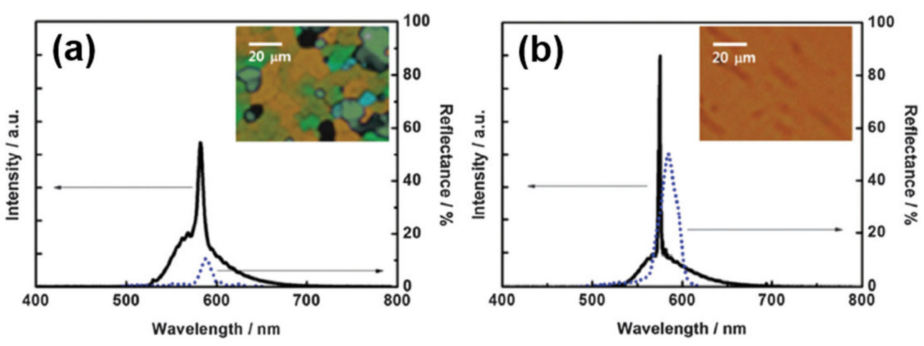
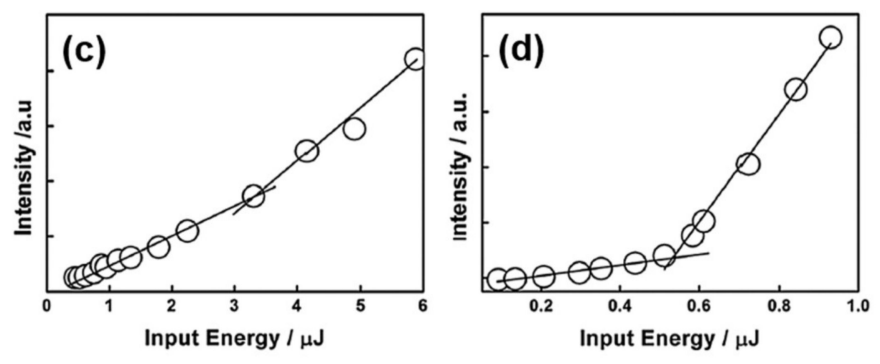

(e)

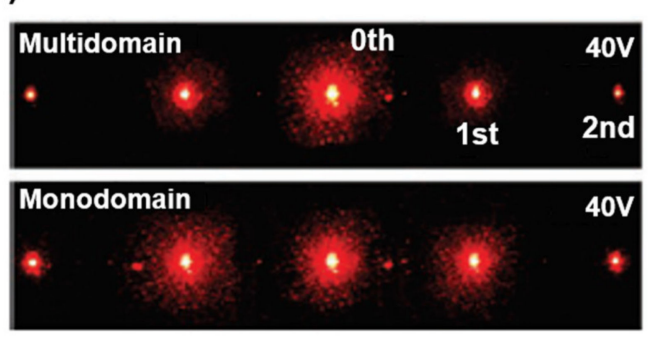

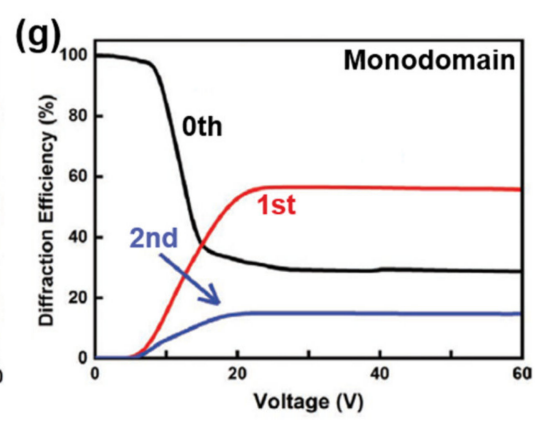

Figure 3. (a,b) Emission spectrum with POM texture (inset) of multidomain BP I (a) and monodomain BP II (b). (c,d) Output intensity with respect to the input energy of multidomain BP I (c) and monodomain BP II (d). (e) Far-field diffraction spots of multidomain (upper) and monodomain (lower) BP I upon applying voltage of $40 \mathrm{~V}$. (f,g) Applied voltage dependent diffraction efficiencies of multidomain (f) and monodomain (g) PSBP I. (a-d) Reproduced with permission from [18]. Copyright 2015, The Royal Society of Chemistry (RSC). (e-g) Reproduced with permission from [44]. Copyright 2020, John Wiley and Sons.

The 3D orientation behavior of the BPs was investigated by observing the phase transitions of the BPs using two types of unidirectionally anchored surfaces [45]. It was revealed that the alignment of BP II obtained by cooling from the isotropic state was extremely sensitive to the surface conditions because different crystal planes, $\mathrm{BP} \mathrm{II}_{(110)}$ for rubbed substrate and $\mathrm{BP} \mathrm{II}_{(100)}$ for photo-aligned substrate, were obtained. Moreover, it was found that BPs show the strong alignment hysteresis of which the orientations of $\mathrm{BP}$ crystals are determined by the preceding structures. For example, BP $\mathrm{I}_{(110)}$ becomes dominant when transited from BP $\mathrm{II}_{(100)}$, whereas BP $\mathrm{I}_{(211)}$ is obtained when transited from BP $\mathrm{II}_{(110)}$. Additionally, BP I obtained from a planar cholesteric liquid crystal (ChLC) showed the (100) crystal plane oriented parallel to the substrate. This result indicates that the orientation of BPs can be easily directed by appropriately controlling the preceding LC structures.

A facile method to pattern the crystallographic orientation of BPs was developed by a photo-alignment technology [46]. A micropattern that exhibits alternate uniform (exposed region) and random (unexposed region) crystal orientations of BP II was realized by spatially illuminating a linear polarized light through a binary photo-mask as shown in Figure $4 \mathrm{a}$. The BP crystals can be deformed by electric field treatment due to the external stimuli-responsivity of LCs, enabling the Bragg reflection wavelength to be tuned with a fast response time. Figure $4 \mathrm{~b}$ shows the far-field diffraction spot from the micropatterned BP II when applying voltage of $0 \mathrm{~V}$ (Figure $4 \mathrm{~b}(\mathrm{i}, \mathrm{ii})$ ) and $3.5 \mathrm{~V}$ (Figure $4 \mathrm{~b}$ (iii,iv)). Before applying electric field, the diffraction pattern appears when illuminated by light with wavelength of $532 \mathrm{~nm}$ that corresponds to the Bragg reflection band of the device (Figure $4 \mathrm{bi}$ ). On the other hand, upon application of electric voltage, the Bragg reflection band red-shifts, 
resulting in the diffraction pattern appearing at a longer wavelength region. Moreover, the rewritable capability of the azo-based alignment layer enables the micropattern to be switched to other patterns by illuminating light through different binary photo-masks and electric field treatment, as shown in Figure 4c. This method gives the capability to create various far-field diffraction patterns such as gratings and vortex beams through the binary reflection amplitude modulation.
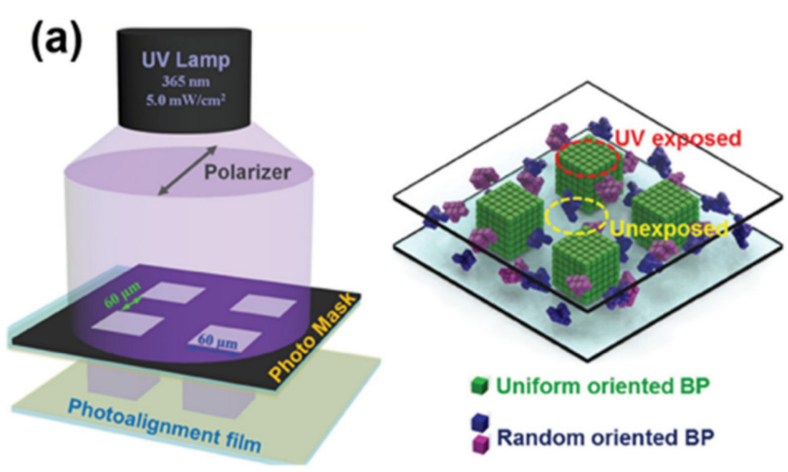

(b)
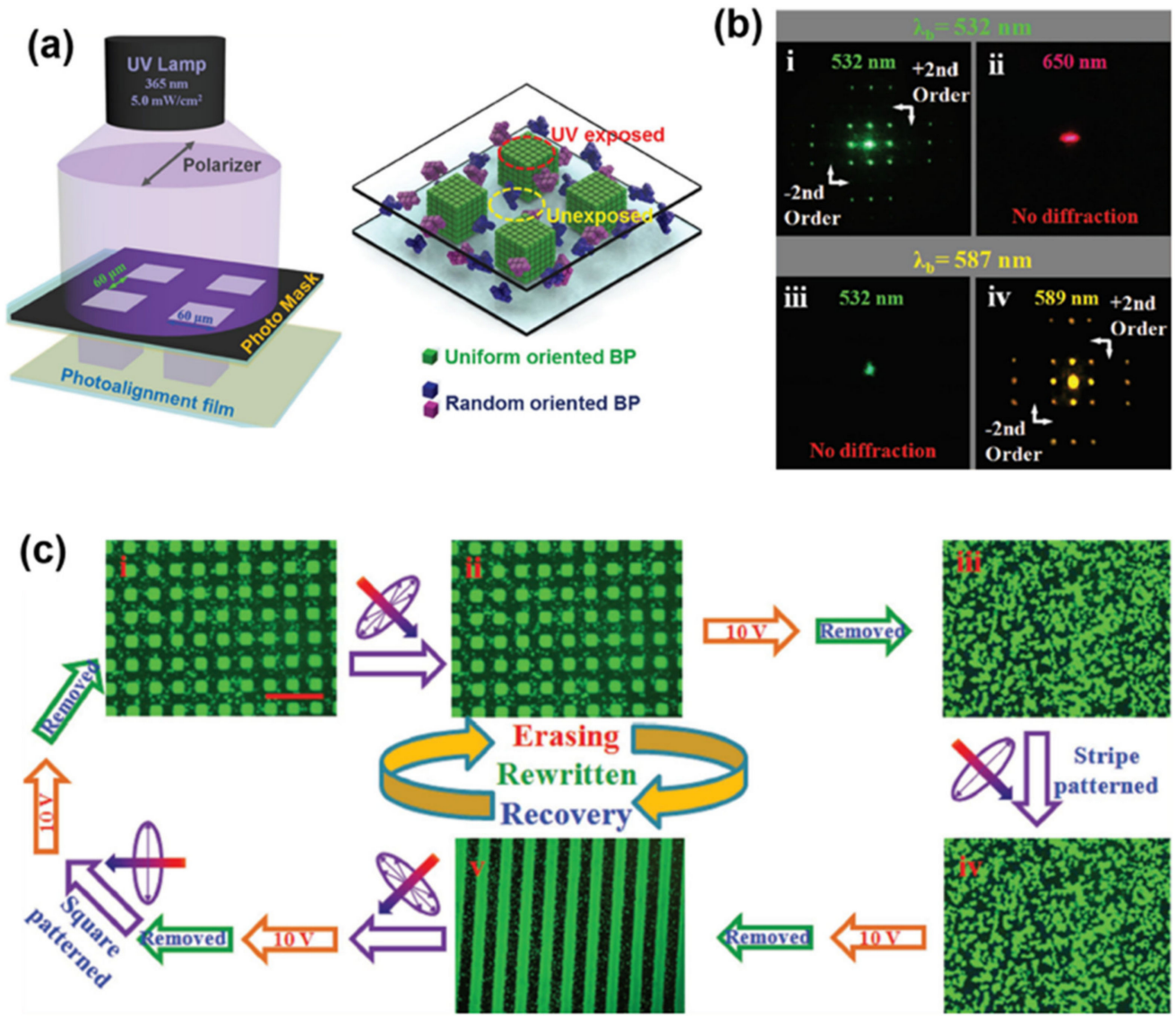

Figure 4. (a) Micropatterned BPs fabricated by illuminating ultraviolet (UV) light through a binary photo-mask. The region where UV light was illuminated has a uniform crystal orientation, whereas other regions have random oriented BP crystals. (b) External stimuli-responsive far-field diffraction patterns. (c) Rewritable capability of the micropatterns by illuminating non-polarized UV light and treating electric field treatment (ii,iii), and by illuminating UV light through a striped photo-mask and treating electric field treatment (iv,v). Reproduced with permission from [46]. Copyright 2017, John Wiley and Sons.

Otón et al. developed a method to predict the crystal orientation of monodomain BPs on a rubbed surface by controlling the precursor materials formulation [47]. They discovered that the crystal orientation of monodomain BPs depends on the lattice constant of BPs, and thus, the tailoring of crystal orientation can be induced by knowing the chiral twisting power of the system and adjusting the concentration of doped chiral dopants.

\subsection{Application of Electric Field}

The treatment of a vertical AC field in BPs enabled monodomain BPs to be obtained without the need of alignment layers on a substrate [48]. By applying and removing the electric field, the (110) crystal plane became dominant parallel to the substrate, resulting a monodomain-like BP textures with uniform reflection colors (Figure 5). This approach allows the crystal orientation to be maintained even when the temperature is raised to an isotropic state and then cooled to BP I. Chen et al. discussed a mechanism of the 
field-reorientation process of BP crystals by repeatedly applying and removing a vertical electric field [49]. When the field was applied, BPs were phase-transited to focal conic and homeotropic state. As the field was removed from the sample, the focal conic state transited to a BP with a uniform reflection color corresponding to the (110) crystal plane, whereas the homeotropic state turned into the BP $\mathrm{I}_{(200)}$ and BP $\mathrm{I}_{(211)}$. When the field was applied again, the portion of the focal conic state became wider than the first step, resulting in the (110) crystal plane to be dominant over a larger area as the field was removed. Thus, by repeating the electric field treatment, the growth of the different crystal planes was suppressed and only a monodomain $B P \mathrm{I}_{(110)}$ was obtained. The monodomain BPs showed higher reflectivity with narrower bandwidth and less hysteresis compared to multidomain BPs (Figure $5 b$ ).

(a)

\section{Before applying $E$ field}

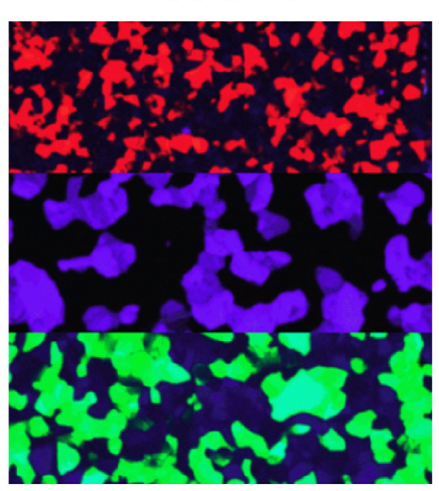

\section{After removing $E$ field}

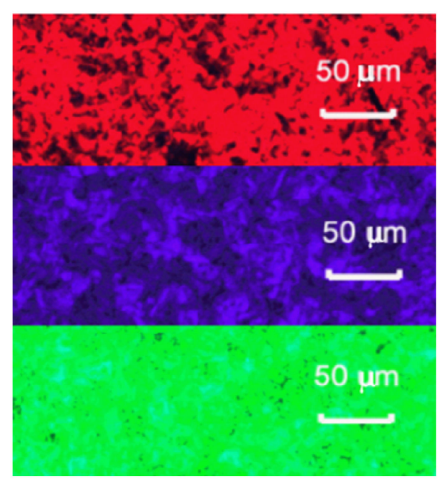

(b)

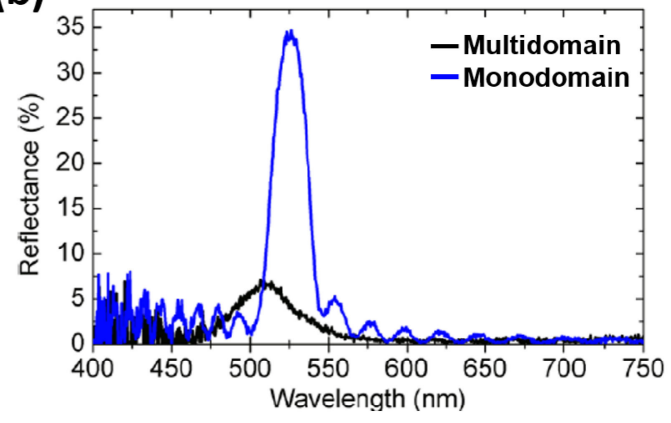

Figure 5. (a) POM textures of BP I before applying electric field (left) and after removing electric field (right). (b) Reflection spectra of multidomain and monodomain BPs. Reproduced with permission from [48], Copyright 2013, American Institute of Physics.

A field-induced bistable effect in the crystal orientation of BPs and its phase-transition mechanism were revealed using a host LC mixture with a negative dielectric anisotropy [50]. Obtaining BP I by removing the electric field from the field-induced planar ChLC state enabled a BP $\mathrm{I}_{(200)}$ to become dominant, because the helical structure of the planar ChLC has a similar structure, with the DTC aligned perpendicular to the substrate. On the other hand, a BP $\mathrm{I}_{(110)}$ was obtained when the field was removed from the field-induced flow state that was similar to Helfrich deformation induced by the electrohydrodynamic effect. Because the electrohydrodynamic effect increases the temperature of the sample, the field-induced flow state is phase-transited to the BP $\mathrm{I}_{(110)}$ in a similar manner to the BP I obtained from the BP II by cooling the temperature.

A monodomain BP was obtained by initiating and growing the nuclei of BP crystals during an application of electric field [51]. When the electric field is applied at an isotropic state and the temperature of the sample is decreased to initiate the nucleation of BP crystals, heterogeneous nucleation plays a dominant role for the growth of BP crystals, while the homogeneous nucleation is suppressed. The nuclei generated through the heterogeneous process gradually grow as the temperature decreases, providing monodomain BPs in the entire region where the field is applied. This phenomenon becomes dominant upon high field intensity at a certain field frequency.

The orientation control using electric field treatment allows us to rapidly obtain monodomain BPs over a large area, but many grain boundaries still exist between the domains. Moreover, those methods can only enable a crystallographic axis of BPs to be oriented perpendicular to the substrate (crystal planes), and other axes still remain random. 


\subsection{Use of Nanopatterned Substrates}

A new strategy to obtain single-crystal BPs was recently proposed by directing the self-assembly process of BPs using lithographically nanopatterned substrates $[52,53]$. The preferred orientation of the LC molecules descripted by the scalar order parameter $(S)$ was theoretically considered for each crystallographic orientation of BPs to minimize the free energy density on a substrate. Based on simulation results, various nanopatterned templates that periodically imposed the homeotropic $(\mathrm{H})$ and planar $(\mathrm{P})$ alignments were designed (Figure $6 \mathrm{a}-\mathrm{c}$ ). For example, a striped pattern of which the $\mathrm{H}$ and $\mathrm{P}$ alignments exist alternately and the sum of the width in two regions is equal to the lattice constant of BP II was employed to obtain a single-domain BP $\mathrm{II}_{(100)}$ over an entire patterned area without grain boundaries (Figure $6 \mathrm{~d}$ ). The crystal orientations of the BP $\mathrm{II}_{(110)}$ and $\mathrm{BP} \mathrm{II}_{(111)}$ were also demonstrated using different nanopatterned templates designed in the same manner, as shown in Figure $6 \mathrm{~b}, \mathrm{c}$, respectively. This method enables the single-crystal BPs to be grown up to a device thickness of approximately $20 \mu \mathrm{m}$ [54]. The single-crystal BP II showed a single and sharp reflection peak compared to polycrystalline BPs that exhibit multiple reflection peaks due to the nonuniform lattice orientations.

The nanopatterned templates were also employed to investigate the phase transition of BPs between two different crystallographic symmetries of single-crystal BP $\mathrm{I}_{(110)}$ and BP II ${ }_{(100)}[55]$. It was revealed that the transition from the single-crystal BP II $(100)$ to BP $\mathrm{I}_{(110)}$ could be regarded as martensitic-like transformation. When the $\mathrm{BP}$ reaches the transition temperature of $\mathrm{BP} \mathrm{II}_{(100)}-\mathrm{BP}_{(110)}$, the DTCs are reorganized through the local reorientation of the LC molecules, resulting in a smooth reconfiguration of the dislocation lines in a diffusionless manner on a sub-micrometer scale. This process has a little thermal hysteresis, enabling the BP crystals to be simultaneously grown in the entire patterned region. This result gives an opportunity to investigate the crystal nucleation of BPs and control the crystal growth dynamics in soft materials.

A strategy to precisely design the grain boundaries between two single-crystal BPs that have different crystal orientations, referred to as soft heteroepitaxy, was investigated by using the lithographically nanopatterned templates [56]. A C-shaped template of which the inside had a striped pattern responsible for the orientation of $\mathrm{BP} \mathrm{II}_{(100)}$ and the outside had a rectangular pattern responsible for $\mathrm{BP} \mathrm{II}_{(110)}$ was employed to observe the grain boundaries between those of crystal planes. Despite the curved geometry, the grain boundary was stabilized and elaborately sculpted on a scale similar to the lattice constant of BPs. This method was applied to fabricating a 2D diffraction grating as an external stimuli-responsive optical device. Because of the precisely sculpted grain boundaries, the signal-to-noise ratio (SNR) of the far-field diffraction patterns was significantly improved compared to the 2D diffraction gratings fabricated by controlling the crystal orientation using photo-alignment technology [46]. This work accelerates the investigation in the nucleation and growth of $\mathrm{BP}$ crystals between liquid-liquid interfaces, and the fabrication of intelligent photonic crystal devices. 

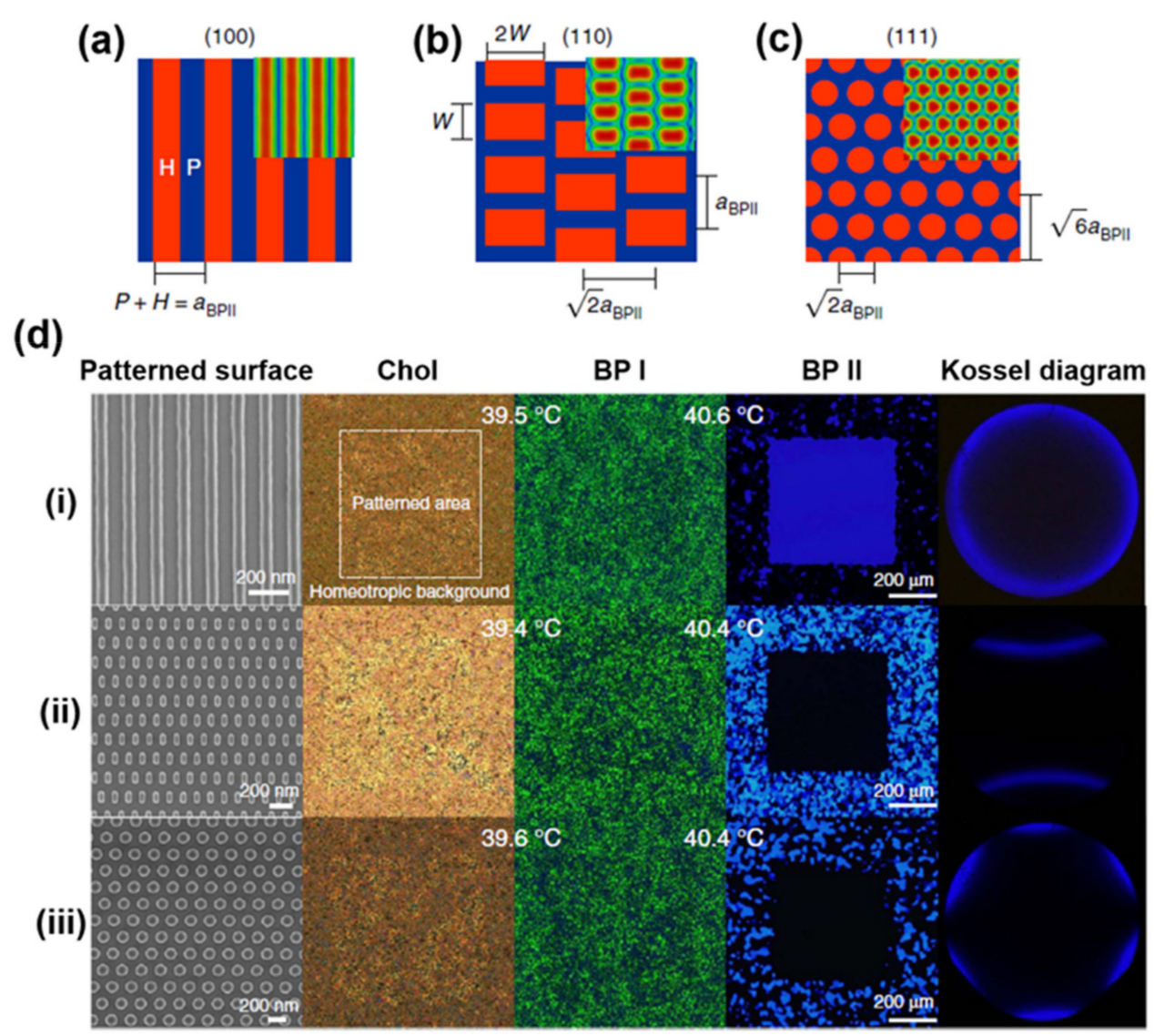

Figure 6. $(\mathrm{a}-\mathrm{c})$ Designs of lithographically nanopatterned templates for the orientation of BP $\mathrm{II}_{(100)}$ (striped pattern), $\mathrm{BP} \mathrm{II}(110)$ (rectangular pattern), and BP II ${ }_{(111)}$ (circular pattern), with insets of $S$-map representing the preferred molecular orientation of LCs on the templates. (d) The scanning electron microscope image of nanopatterned templates, the POM texture of BPs, and Kossel diagrams corresponding to the BP II region, on the different templates, such as (i) striped pattern, (ii) rectangular pattern, and (iii) circular pattern. Reproduced with permission from [52]. Copyright 2017, Nature Publishing Group.

\section{Azimuthal Orientation-Controlling Technologies of BPs}

The aforementioned approaches mainly focus on obtaining monodomain-like BP textures with a uniform crystal plane orientation. However, it is required to simultaneously manipulate two independent crystal axes of BPs to uniquely define the 3D crystal orientation on a substrate. Recent studies to investigate the grain boundaries between different orientations of BP crystals [56] or realize reflective volume holograms through the Bragg-Berry (BB) effect [36] have emphasized the importance of simultaneously controlling the crystal plane and azimuthal orientation of BPs. In the following, we discuss strategies to uniquely define 3D orientation of BPs on a substrate, with its mechanisms and perspective photonic applications.

The highly ordered structures of BPs with a periodicity of a few hundred nm give rise to a unique $\mathrm{CP}$-selective Bragg reflection in visible light region. With the external stimuliresponsivity of LCs, many tunable devices have been proposed by modulating either the intensity or wavelength of Bragg reflected light from BPs. On the other hand, it was recently found that a $0-2 \pi$ full wavefront control of reflected light can be attained by azimuthally rotating $\mathrm{BP}$ crystals on a substrate, which is referred to as the $\mathrm{BB}$ effect. The simulations revealed that both $B P \mathrm{I}_{(110)}$ and $B P \mathrm{II}_{(100)}$ show the $\mathrm{BB}$ effect in the same manner [36]. The theoretical prediction was experimentally demonstrated by spatially rotating BP II crystals on a substrate through photo-alignment technology. The orientation of the BP $\mathrm{II}_{(100)}$ was manipulated with a [100] axis (azimuthal orientation) to follow a predesigned surface 
anchoring and another [100] axis (crystal plane orientation) was aligned along the substrate normal. Whether the wavefront was indeed being controlled was confirmed through the observation of an interferometric microscope image. This result demonstrates that a full $0-2 \pi$ phase-only modulation with a CP selectivity provides opportunities for advanced DOEs, such as flat deflectors, lenses, and holograms.

While the azimuthal orientation of the BP $\mathrm{II}_{(100)}$ can be made to follow the imprinted surface anchoring on a substrate, it had still remained a challenge to attain the tailored azimuthal orientation of BP I on a substrate. Recently, a strategy to control the azimuthal orientation of BP I was proposed using a field-induced directed self-assembly process [37]. At a BP $\mathrm{I}_{(211)}$ prepared by cooling from the isotropic state, an electric field was applied to unwind the twist structures and gradually reduced to reorient the BP crystals. A BP I with a different reflection color compared to the initial BP $\mathrm{I}_{(211)}$ was obtained through the phase transitions of homeotropic-chiral nematic-BP X-BP I (Figure 7a). The two-fold symmetry of the Kossel diagrams revealed that the field-reoriented BP I has a (110) crystal plane parallel to the substrate (Figure $7 \mathrm{~b}$ ). Further, the Kossel patterns were rotated with a step of $45^{\circ}$, indicating that the azimuthal orientation ( [001] crystal axis) was also controlled to follow the imprinted surface anchoring. These results showed that two crystal axes of BP I can be simultaneously controlled along the predesigned directions. The mechanism was investigated by observing the two field-induced intermediate phases, chiral nematic and BP X. The chiral nematic phase plays a key role to control the [001] axis along the predesigned direction. The field-induced chiral nematic was composed of the uniformly lying helix (ULH) with the helical axis perpendicular to the surface anchoring with LC molecules aligned along the surface anchoring on a substrate, which is a similar structure to a DTC. An additional twist of the LC molecules to create the DTC from the ULH was formed perpendicular to those of the surface anchoring and helical axis, making the DTC to be aligned along the surface anchoring. On the other hand, the field-induced BP X was responsible for the (110) crystal plane orientation of the field-induced BP I. This result was consistent with the simulation results which revealed that BP X can be obtained via continuous reorganization from BP $\mathrm{I}_{(110)}[57,58]$.

The field-assisted directed self-assembly process was applied to a fabrication of a phase-only BP-based hologram based on the BB effect. The unique 3D chiral structure of $\mathrm{BPs}$ gave a capability to play back the encoded light information for only a CP light in a wide range of incident angles (Figure 7c), which were distinct from the holograms realized by ChLCs $[59,60]$. The holograms were expected to have significantly high light-conversion efficiency compared to binary-amplitude DOEs made by spatial control of only crystal plane orientation of BPs $[12,46,56]$. 

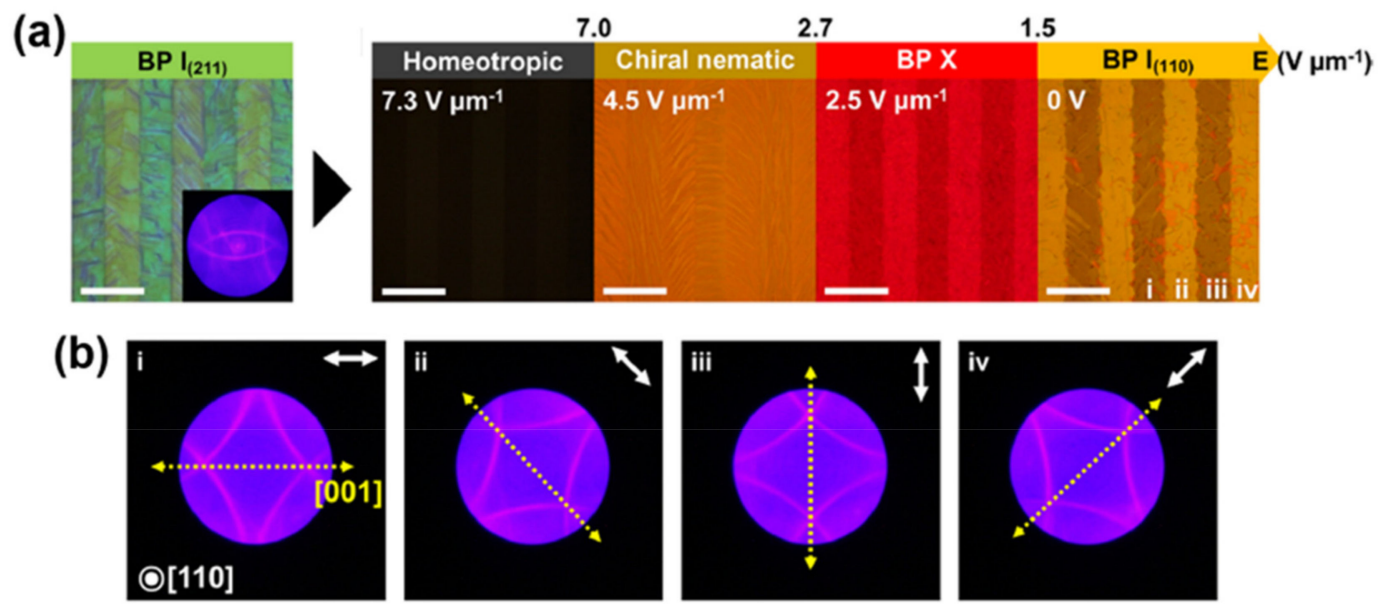

(c)
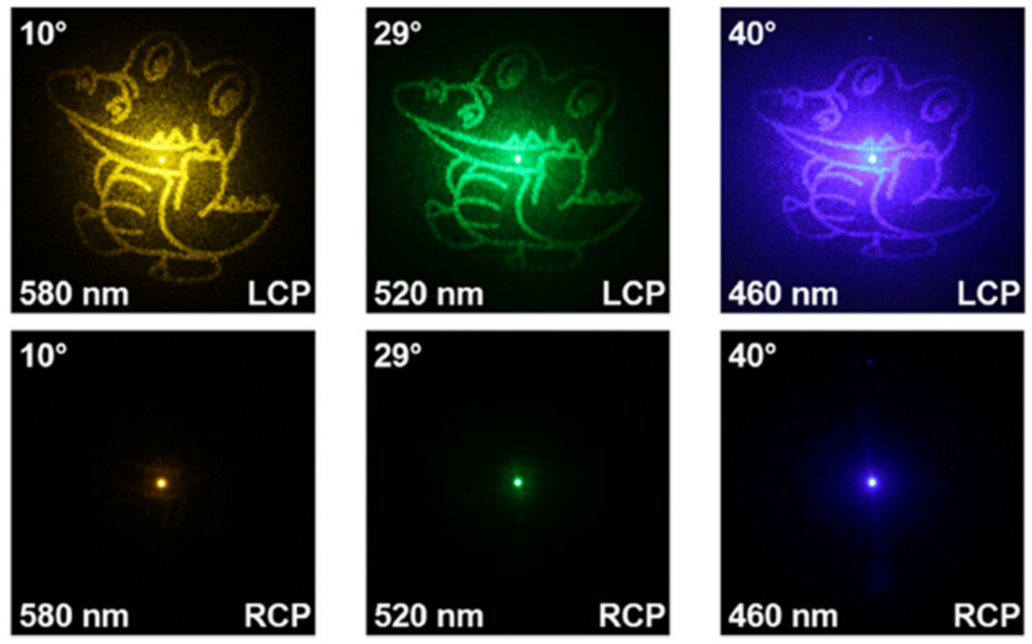

Figure 7. (a) The evolution of POM images of the field-induced phase transition when the field is applied to $\mathrm{H}$ phase and gradually reduced. (b) Kossel diagrams of the field-induced BP I at each striped region marked as the Roman numeral in (a). The double-sided white arrows and the yellow arrows show the imprinted surface anchoring in each region and the [001] crystal axis of the field-induced BP I. (c) Holograms reconstructed at incident angles of $10^{\circ}, 29^{\circ}$, and $40^{\circ}$ for left (L) and right (R) CP light illumination with wavelength of $580 \mathrm{~nm}, 520 \mathrm{~nm}$, and $460 \mathrm{~nm}$, respectively. Reproduced with permission from [37]. Copyright 2021, Nature Publishing Group.

\section{Discussion}

In this paper, we have drawn recent research progress to direct the 3D orientation of two types of cubic BPs: body-centered BP I and simple cubic BP II, along the predesigned directions on a substrate. Various strategies have emerged to manipulate either the crystal plane and azimuthal orientation of BPs, such as directing the self-assembly process and external field treatments. Controlling the 3D orientation of the BPs is one of the important steps in both science and industry fields, because it provides a degree of freedom to manipulate the light propagating through the medium, giving tailored optical characteristics in a thickness of a sub-micrometer scale. By combining the external stimuli-responsivity of BPs, various photonic devices that have not been discovered yet can be developed with high-levels and integrated optical functionalities. For example, we expect that 3D emission direction-controllable photonic crystal lasers can be achieved by introducing a slanted crystal plane orientation and controlling its periodicity [61]. Moreover, BPs have a great potential to fabricate flexible films by photo-polymerizing the LC materials [62-65], making them applicable to wearable devices such as smart-glasses. Although there still remain several questions that should be addressed such as the orientation behavior of the 
LC molecules in grain boundaries, it is manifest that BPs have a great deal of potential in various scientific research field and the development of practical devices in a wide range from displays to holographic optical elements.

Author Contributions: Writing-original draft, S.C.; writing-review and editing, S.C. and M.O.; supervision, M.O. Funding acquisition, M.O. All authors have read and agreed to the published version of the manuscript.

Funding: This work was supported by the JSPS KAKENHI (20H00391, and 20H04672) and the JSPS Core-to-Core Program A., Advanced Research Networks.

Data Availability Statement: This study did not report any data.

Conflicts of Interest: The authors declare no conflict of interest.

\section{References}

1. Kikuchi, H. Liquid crystalline blue phases. Struct. Bond. 2007, 128, 99-117.

2. Higashiguchi, K.; Yasui, K.; Kikuchi, H. Direct observation of polymer-stabilized blue phase I structure with confocal laser scanning microscope. J. Am. Chem. Soc. 2008, 130, 6326-6327. [CrossRef]

3. Tanaka, S.; Yoshida, H.; Kawata, Y.; Kuwahara, R.; Nishi, R.; Ozaki, M. Double-twist cylinders in liquid crystalline cholesteric blue phases observed by transmission electron microscopy. Sci. Rep. 2015, 5, 16180. [CrossRef] [PubMed]

4. Yoshida, H.; Anucha, K.; Ogawa, Y.; Kawata, Y.; Ozaki, M.; Fukuda, J.-I.; Kikuchi, H. Bragg reflection band width and optical rotatory dispersion of cubic blue-phase liquid crystals. Phys. Rev. E 2016, 94, 042703. [CrossRef]

5. Belyakov, V.; Demikhov, E.; Dmitrienko, V.; Dolganov, V. Optical activity, transmission spectra, and structure of blue phases of liquid crystals. J. Exp. Theor. Phys. 1985, 89, 2035-2051.

6. Hur, S.T.; Lee, B.R.; Gim, M.J.; Park, K.W.; Song, M.H.; Choi, S.W. Liquid-Crystalline Blue Phase Laser with Widely Tunable Wavelength. Adv. Mater. 2013, 25, 3002-3006. [CrossRef]

7. Wang, L.; Li, Q. Stimuli-directing self-organized 3D liquid-crystalline nanostructures: From materials design to photonic applications. Adv. Funct. Mater. 2016, 26, 10-28. [CrossRef]

8. Chen, C.-W.; Li, C.-C.; Jau, H.-C.; Yu, L.-C.; Hong, C.-L.; Guo, D.-Y.; Wang, C.-T.; Lin, T.-H. Electric field-driven shifting and expansion of photonic band gaps in 3D liquid photonic crystals. ACS Photonics 2015, 2, 1524-1531. [CrossRef]

9. Du, X.-W.; Hou, D.-S.; Li, X.; Sun, D.-P.; Lan, J.-F.; Zhu, J.-L.; Ye, W.-J. Symmetric continuously tunable photonic band gaps in blue-phase liquid crystals switched by an alternating current field. ACS Appl. Mater. Interfaces 2019, 11, 22015-22020. [CrossRef] [PubMed]

10. Guo, D.-Y.; Chen, C.-W.; Li, C.-C.; Jau, H.-C.; Lin, K.-H.; Feng, T.-M.; Wang, C.-T.; Bunning, T.J.; Khoo, I.C.; Lin, T.-H. Reconfiguration of three-dimensional liquid-crystalline photonic crystals by electrostriction. Nat. Mater. 2020, 19, 94-101. [CrossRef]

11. Xu, X.; Liu, Z.; Liu, Y.; Zhang, X.; Zheng, Z.; Luo, D.; Sun, X. Electrically switchable, hyper-reflective blue phase liquid crystals films. Adv. Opt. Mater. 2018, 6, 1700891. [CrossRef]

12. Zhou, K.; Bisoyi, H.K.; Jin, J.Q.; Yuan, C.L.; Liu, Z.; Shen, D.; Lu, Y.Q.; Zheng, Z.G.; Zhang, W.; Li, Q. Light-Driven Reversible Transformation between Self-Organized Simple Cubic Lattice and Helical Superstructure Enabled by a Molecular Switch Functionalized Nanocage. Adv. Mater. 2018, 30, 1800237. [CrossRef]

13. Chen, X.; Wang, L.; Li, C.; Xiao, J.; Ding, H.; Liu, X.; Zhang, X.; He, W.; Yang, H. Light-controllable reflection wavelength of blue phase liquid crystals doped with azobenzene-dimers. Chem. Commun. 2013, 49, 10097-10099. [CrossRef]

14. Yan, J.; Wu, S.-T.; Cheng, K.-L.; Shiu, J.-W. A full-color reflective display using polymer-stabilized blue phase liquid crystal. Appl. Phys. Lett. 2013, 102, 081102. [CrossRef]

15. Yokoyama, S.; Mashiko, S.; Kikuchi, H.; Uchida, K.; Nagamura, T. Laser emission from a polymer-stabilized liquid-crystalline blue phase. Adv. Mater. 2006, 18, 48-51. [CrossRef]

16. Cao, W.; Munoz, A.; Palffy-Muhoray, P.; Taheri, B. Lasing in a three-dimensional photonic crystal of the liquid crystal blue phase II. Nat. Mater. 2002, 1, 111-113. [CrossRef] [PubMed]

17. Petriashvili, G.; Chanishvili, A.; Zurabishvili, T.; Chubinidze, K.; Ponjavidze, N.; De Santo, M.P.; Bruno, M.D.L.; Barberi, R. Temperature tunable omnidirectional lasing in liquid crystal blue phase microspheres. OSA Contin. 2019, 2, 3337-3342. [CrossRef]

18. Kim, K.; Hur, S.-T.; Kim, S.; Jo, S.-Y.; Lee, B.R.; Song, M.H.; Choi, S.-W. A well-aligned simple cubic blue phase for a liquid crystal laser. J. Mater. Chem. C 2015, 3, 5383-5388. [CrossRef]

19. Bukusoglu, E.; Wang, X.; Martinez-Gonzalez, J.A.; de Pablo, J.J.; Abbott, N.L. Stimuli-responsive cubosomes formed from blue phase liquid crystals. Adv. Mater. 2015, 27, 6892-6898. [CrossRef]

20. Martínez-González, J.A.; Zhou, Y.; Rahimi, M.; Bukusoglu, E.; Abbott, N.L.; de Pablo, J.J. Blue-phase liquid crystal droplets. Proc. Natl. Acad. Sci. USA 2015, 112, 13195-13200. [CrossRef]

21. Chen, Y.-H.; Wang, C.-T.; Yu, C.-P.; Lin, T.-H. Polarization independent Fabry-Pérot filter based on polymer-stabilized blue phase liquid crystals with fast response time. Opt. Express 2011, 19, 25441-25446. [CrossRef] [PubMed]

22. Chen, H.-Y.; Tu, H.-Y. Optical polarization states of a liquid-crystal blue phase II. OSA Contin. 2019, 2, 478-485. [CrossRef] 
23. Yan, J.; Li, Y.; Wu, S.-T. High-efficiency and fast-response tunable phase grating using a blue phase liquid crystal. Opt. Lett. 2011, 36, 1404-1406. [CrossRef] [PubMed]

24. Ge, S.-J.; Ji, W.; Cui, G.-X.; Wei, B.-Y.; Hu, W.; Lu, Y.-Q. Fast switchable optical vortex generator based on blue phase liquid crystal fork grating. Opt. Mater. Express 2014, 4, 2535-2541. [CrossRef]

25. Kikuchi, H.; Yokota, M.; Hisakado, Y.; Yang, H.; Kajiyama, T. Polymer-stabilized liquid crystal blue phases. Nat. Mater. 2002, 1, 64-68. [CrossRef] [PubMed]

26. Castles, F.; Day, F.; Morris, S.; Ko, D.; Gardiner, D.; Qasim, M.; Nosheen, S.; Hands, P.; Choi, S.; Friend, R. Blue-phase templated fabrication of three-dimensional nanostructures for photonic applications. Nat. Mater. 2012, 11, 599-603. [CrossRef]

27. Yoshida, H.; Tanaka, Y.; Kawamoto, K.; Kubo, H.; Tsuda, T.; Fujii, A.; Kuwabata, S.; Kikuchi, H.; Ozaki, M. Nanoparticle-stabilized cholesteric blue phases. Appl. Phys. Express 2009, 2, 121501. [CrossRef]

28. Draude, A.P.; Kalavalapalli, T.Y.; Iliut, M.; McConnell, B.; Dierking, I. Stabilization of liquid crystal blue phases by carbon nanoparticles of varying dimensionality. Nanoscale Adv. 2020, 2, 2404-2409. [CrossRef]

29. Hur, S.-T.; Gim, M.-J.; Yoo, H.-J.; Choi, S.-W.; Takezoe, H. Investigation for correlation between elastic constant and thermal stability of liquid crystalline blue phase I. Soft Matter 2011, 7, 8800-8803. [CrossRef]

30. Yoshizawa, A.; Sato, M.; Rokunohe, J. A blue phase observed for a novel chiral compound possessing molecular biaxiality. J. Mater. Chem. 2005, 15, 3285-3290. [CrossRef]

31. Ojima, M.; Noma, T.; Asagi, H.; Fujii, A.; Ozaki, M.; Kikuchi, H. Pinning effect of mixed cellulose ester membrane on appearance of cholesteric blue phases. Appl. Phys. Express 2009, 2, 021502. [CrossRef]

32. Noma, T.; Ojima, M.; Asagi, H.; Kawahira, Y.; Fujii, A.; Ozaki, M.; Kikuchi, H. Effects of polymer network surfaces on expansion of cholesteric blue phases temperature. e-J. Surf. Sci. Nanotechnol. 2008, 6, 17-20. [CrossRef]

33. Lin, J.-D.; Ho, Y.-L.D.; Chen, L.; Lopez-Garcia, M.; Jiang, S.-A.; Taverne, M.P.; Lee, C.-R.; Rarity, J.G. Microstructure-stabilized blue phase liquid crystals. ACS Omega 2018, 3, 15435-15441. [CrossRef] [PubMed]

34. Coles, H.J.; Pivnenko, M.N. Liquid crystal 'blue phases' with a wide temperature range. Nature 2005, 436, 997-1000. [CrossRef] [PubMed]

35. Yang, J.; Zhao, W.; He, W.; Yang, Z.; Wang, D.; Cao, H. Liquid crystalline blue phase materials with three-dimensional nanostructures. J. Mater. Chem. C 2019, 7, 13352-13366. [CrossRef]

36. Yoshida, H.; Kobashi, J. Flat optics with cholesteric and blue phase liquid crystals. Liq. Cryst. 2016, 43, 1909-1919. [CrossRef]

37. Cho, S.; Takahashi, M.; Fukuda, J.-I.; Yoshida, H.; Ozaki, M. Directed self-assembly of soft 3D photonic crystals for holograms with omnidirectional circular-polarization selectivity. Commun. Mater. 2021, 2, 39. [CrossRef]

38. Chen, H.-S.; Lin, Y.-H.; Wu, C.-H.; Chen, M.; Hsu, H.-K. Hysteresis-free polymer-stabilized blue phase liquid crystals using thermal recycles. Opt. Mater. Express 2012, 2, 1149-1155. [CrossRef]

39. Chen, C.-W.; Hou, C.-T.; Li, C.-C.; Jau, H.-C.; Wang, C.-T.; Hong, C.-L.; Guo, D.-Y.; Wang, C.-Y.; Chiang, S.-P.; Bunning, T.J. Large three-dimensional photonic crystals based on monocrystalline liquid crystal blue phases. Nat. Commun. 2017, 8, 727. [CrossRef] [PubMed]

40. Nayek, P.; Jeong, H.; Park, H.R.; Kang, S.-W.; Lee, S.H.; Park, H.S.; Lee, H.J.; Kim, H.S. Tailoring monodomain in blue phase liquid crystal by surface pinning effect. Appl. Phys. Express 2012, 5, 051701. [CrossRef]

41. Kim, K.; Kim, S.; Jo, S.-Y.; Choi, S.-W. A monodomain-like liquid-crystalline simple cubic blue phase II. J. Inf. Disp. 2015, 16, 155-160. [CrossRef]

42. Oton, E.; Netter, E.; Nakano, T.; Inoue, F. Monodomain blue phase liquid crystal layers for phase modulation. Sci. Rep. 2017, 7, 44575. [CrossRef]

43. Jo, S.-Y.; Jeon, S.-W.; Kim, B.-C.; Bae, J.-H.; Araoka, F.; Choi, S.-W. Polymer stabilization of liquid-crystal blue phase II toward photonic crystals. ACS Appl. Mater. Interfaces 2017, 9, 8941-8947. [CrossRef] [PubMed]

44. Manda, R.; Pagidi, S.; Heo, Y.J.; Lim, Y.J.; Kim, M.S.; Lee, S.H. Polymer-Stabilized Monodomain Blue Phase Diffraction Grating. Adv. Mater. Interfaces 2020, 7, 1901923. [CrossRef]

45. Takahashi, M.; Ohkawa, T.; Yoshida, H.; Fukuda, J.-I.; Kikuchi, H.; Ozaki, M. Orientation of liquid crystalline blue phases on unidirectionally orienting surfaces. J. Phys. D Appl. Phys. 2018, 51, 104003. [CrossRef]

46. Zheng, Z.G.; Yuan, C.L.; Hu, W.; Bisoyi, H.K.; Tang, M.J.; Liu, Z.; Sun, P.Z.; Yang, W.Q.; Wang, X.Q.; Shen, D. Light-patterned crystallographic direction of a self-organized 3d soft photonic crystal. Adv. Mater. 2017, 29, 1703165. [CrossRef] [PubMed]

47. Otón, E.; Yoshida, H.; Morawiak, P.; Strzeżysz, O.; Kula, P.; Ozaki, M.; Piecek, W. Orientation control of ideal blue phase photonic crystals. Sci. Rep. 2020, 10, 10148. [CrossRef]

48. Chen, Y.; Wu, S.-T. Electric field-induced monodomain blue phase liquid crystals. Appl. Phys. Lett. 2013, 102, 171110. [CrossRef]

49. Chen, M.; Lin, Y.-H.; Chen, H.-S.; Chen, H.-Y. Electrically assisting crystal growth of blue phase liquid crystals. Opt. Mater. Express 2014, 4, 953-959. [CrossRef]

50. Wang, C.-T.; Liu, H.-Y.; Cheng, H.-H.; Lin, T.-H. Bistable effect in the liquid crystal blue phase. Appl. Phys. Lett. 2010, 96, 041106. [CrossRef]

51. Yan, J.; Lin, J.; Li, Q.; Li, R.-Z. Influence of long-lasting electric field on the formation of monodomain polymer stabilized blue phase liquid crystals. J. Appl. Phys. 2019, 125, 024501. [CrossRef]

52. Martínez-González, J.A.; Li, X.; Sadati, M.; Zhou, Y.; Zhang, R.; Nealey, P.F.; De Pablo, J.J. Directed self-assembly of liquid crystalline blue-phases into ideal single-crystals. Nat. Commun. 2017, 8, 15854. [CrossRef] 
53. Li, X.; Martínez-González, J.A.; Park, K.; Yu, C.; Zhou, Y.; de Pablo, J.J.; Nealey, P.F. Perfection in nucleation and growth of blue-phase single crystals: Small free-energy required to self-assemble at specific lattice orientation. ACS Appl. Mater. Interfaces 2019, 11, 9487-9495. [CrossRef] [PubMed]

54. Li, X.; Martinez-Gonzalez, J.A.; de Pablo, J.J.; Nealey, P. Thickness dependence of forming single crystal by liquid-crystalline blue phase on chemically patterned surface. SPIE 2018, 10555, 1055514.

55. Li, X.; Martínez-González, J.A.; Hernández-Ortiz, J.P.; Ramírez-Hernández, A.; Zhou, Y.; Sadati, M.; Zhang, R.; Nealey, P.F.; De Pablo, J.J. Mesoscale martensitic transformation in single crystals of topological defects. Proc. Natl. Acad. Sci. USA 2017, 114, 10011-10016. [CrossRef] [PubMed]

56. Li, X.; Martínez-González, J.A.; Guzmán, O.; Ma, X.; Park, K.; Zhou, C.; Kambe, Y.; Jin, H.M.; Dolan, J.A.; Nealey, P.F. Sculpted grain boundaries in soft crystals. Sci. Adv. 2019, 5, eaax9112. [CrossRef]

57. Alexander, G.; Marenduzzo, D. Cubic blue phases in electric fields. Europhys. Lett. 2008, 81, 66004. [CrossRef]

58. Alexander, G.; Yeomans, J. Numerical results for the blue phases. Liq. Cryst. 2009, 36, 1215-1227. [CrossRef]

59. Kobashi, J.; Yoshida, H.; Ozaki, M. Polychromatic optical vortex generation from patterned cholesteric liquid crystals. Phys. Rev. Lett. 2016, 116, 253903. [CrossRef]

60. Cho, S.; Ono, M.; Yoshida, H.; Ozaki, M. Bragg-Berry flat reflectors for transparent computer-generated holograms and waveguide holography with visible color playback capability. Sci. Rep. 2020, 10, 8201. [CrossRef]

61. Cho, S.; Yoshida, H.; Ozaki, M. Emission Direction-Tunable Liquid Crystal Laser. Adv. Opt. Mater. 2020, 8, 2000375. [CrossRef]

62. Lin, T.H.; Li, Y.; Wang, C.T.; Jau, H.C.; Chen, C.W.; Li, C.C.; Bisoyi, H.K.; Bunning, T.J.; Li, Q. Red, green and blue reflections enabled in an optically tunable self-organized 3D cubic nanostructured thin film. Adv. Mater. 2013, 25, 5050-5054. [CrossRef]

63. Yang, J.; Liu, J.; Guan, B.; He, W.; Yang, Z.; Wang, J.; Ikeda, T.; Jiang, L. Fabrication and photonic applications of large-domain blue phase films. J. Mater. Chem. C 2019, 7, 9460-9466. [CrossRef]

64. Yang, J.; Zhao, W.; Yang, Z.; He, W.; Wang, J.; Ikeda, T.; Jiang, L. Printable photonic polymer coating based on a monodomain blue phase liquid crystal network. J. Mater. Chem. C 2019, 7, 13764-13769. [CrossRef]

65. Castles, F.; Morris, S.; Hung, J.; Qasim, M.M.; Wright, A.; Nosheen, S.; Choi, S.; Outram, B.; Elston, S.; Burgess, C. Stretchable liquid-crystal blue-phase gels. Nat. Mater. 2014, 13, 817-821. [CrossRef] [PubMed] 